\title{
14 Multiverse Scenarios
}

In keeping with the 'principle of mediocrity' (aka the Copernican principle), let us assume for the time being that there is nothing special about the universe we happen to inhabit, and that there are many other universes. If they exist, these universes are either fully or almost fully disjoint regions of a larger space-time, and hence we have little or no ability to observe or perceive their direct or indirect effects. As we saw earlier, there exist many possible 'places' where these universes could be located: some could be staggered within cracks opened up by the discreteness of space-time, and hence astonishingly nearby, others might be in totally separate, vastly distant regions. Some, as we will soon see, could be both elsewhere and elsewhen, tucked away in unexpected and exotic places.

Let's first review two further mechanisms for the creation of new universes, which could be more productive than the huge virtual mass scenario envisioned earlier, because huge virtual masses could be just too rare to be able to generate many universes (also see the last paragraph of note 41). Better still, one of these mechanisms entails the unexpected resolution of an abiding problem of time in our universe.

One of these two alternative possible mechanisms is known as 'eternal inflation' and is similar to the Tryon scenario, except that it generalizes the productive effect of random quantum fluctuations to other parameters besides instant virtual masses. Its basic mechanism would be that various scalar fields will time and again happen to fluctuate up to a level where the energy of the quantum vacuum becomes punctually abnormally high. A region of space-time will then demonstrably begin swelling up if vacuum energy becomes its predominant energy; over, say, its matter content and/ or its gravity profile. Such up-swellings can separate out and give rise to new distinct universes.

The other mechanism is the generation of new universes through black holes. We saw earlier that Penrose's theorem predicts the formation of a singularity - a rip in space-time at the black hole. But looking more closely at how singularities may form, a group of physicists wondered whether we were missing a small elephant in the room.

This small elephant is that heavier matter particles, such as protons and neutrons (83), do have extra energy in the form of momentum, a factor that had been overlooked in the standard version of relativity. When they interact with one another, these particles create small amounts of a force called torsion. Too small to amount to anything much under normal circumstances, but becomes significant when matter density increases. When matter becomes so dense as to form a black hole, torsion alone becomes enough to stand in the way of the formation of black holes. At the same time, it generates a huge amount of extra energy which must somehow find an outlet. It soon finds it by creating matter in the form of matched pairs of particles of matter and antimatter. Because of random quantum effects, there will always be a small flickering amount of instantaneous imbalance between the instantaneous quantities 
of matter and antimatter. To cut a long story short, such a small amount of imbalance can give rise to no less than a Big Bangs which blows up within a separate space-time that it creates at the instant it bangs. That type of Big Bang comes complete with an early inflation phase, which physicists were earlier at pains to devise ad hoc theories to explain (84): under this scenario, inflation naturally happens owing to the huge repulsive forces born of the torsion force.

Nikodem Poplawski of Indiana University speculates that this scenario of universe formation by black holes may be creating new universes continuously, including from our very own universe, which would then become the parent universe to an evergrowing number of child universes: every time a supernova goes off somewhere in our universe, a new separate universe is born. He surmises that this scenario is testable, because some of the properties of the parent universe would leak through to the children.

This scenario would also solve one of the most abiding mysteries of time. One of the properties that would seep through is ... the apparent arrow of time, when seen at the scale of a whole universe, an arrow as of yet unexplainable any other way: all of the laws of physics are time invariant, which means that they work transparently and equally well with time going forward or backwards (85). As Nikodem Poplawski puts it, "the arrow of cosmic time of a universe is set by the time-asymmetric collapse of matter through the event horizon during its formation." Seen from outside the black hole that gives rise to the new universe, the horizon's formation and all the subsequent processes all occur after ... infinite time!

Anything that happens, from an outsider's viewpoint, inside the barrier of the event horizon takes place after infinite time when measured by the outsider's clock. Thus, all universes born from black holes are for ever in the future of the original universe containing the generating black hole. Likewise, if we were born from a black hole in a parent universe we are for ever in the future of that parent universe, which for us is stuck for ever in an infinite past. Child universes become unmoored from both their original parent universe and the black hole itself, and go on to create and lead their subsequent lives in whole new branches of space-time, inaccessible for ever from the parent universe.

Besides the manifold ways in which separate universes may be born and go on to populate a metaverse, what form or forms can a metaverse take?

According to Max Tegmark et al., there are broadly 4 distinct categories of possible multiverses, each next level up allowing for more diverse universes:

The Level 1 metaverse is a spatially infinite metaverse, housing universes which essentially feature laws of physics similar to our own. They may however have different initial conditions, boundary values and different values of the fundamental constants.

Level 2 is an elaboration on level 1 whereby universes with all kinds of weird laws of physics could exist, as opposed to the more pedestrian equivalents before. 
Level 3 is a metaverse wherein all of the possible potentialities contained within all possible wave functions are all manifested in reality somewhere

Level 4 is the broadest possible metaverse, a weird, hard-to-conceptualize metaverse, where mathematical structures could be so oddly different from ours that we can't imagine them, yet they do happen somewhere or somewhen.

The idea of the possibility of a multiverse has been around for years but until recently had never much caught on. One of the reasons for this understandable reluctance has to be that the metaverse idea has come burdened with strange baggage, understandably widely perceived as unacceptable and far too weird.

Before we go on, we must visit this issue. There is an affirmation put forward by a number of scientists that a Level 1 multiverse would necessarily contain exact copies of all individuals, yourself and of everybody else, somewhere across or within the metaverse. The assertion is that a metaverse, from Level 1 upwards, would inevitably contain an infinity of exact copies of everybody on Earth, as well as an infinity of all manners of near copies. Some have taken the idea to the extreme. Colin Bruce avers, it is to be hoped apocryphally, that he would be ready to commit suicide without a second thought because he just knows that he'd keep living in an exact similar life somewhere else in the metaverse, and that hence suicide as we know it is factually impossible.

But must not science, in the words of Graham Farmelo, be beautiful?

The physicist Joao Magueijo spoke in his 2003 book 'Faster than the Speed of Light', of experiencing the pursuit of science as follows: "When we touch upon certain scientific realities, we physicists undergo adrenalin spikes far more intense than is achievable by any other experience on Earth. After such experiences, all other human activities - drinking, eating, and socializing with friends - become miserable and dull in comparison. Therein lies the reason why all true scientists are asocial, and tend to become veritable social suicides.” Many other scientists, such as Murray Gell-Mann and others, have echoed these words.

And would not an endless metaverse, wherein an infinity of exact as well as near-exact copies of oneself in all conceivable shadings would hang around in identical and near-identical duplicate lives, be uncommonly ugly? Can a science that accommodates exact copies of yourself somewhere, leading exact and nearly exact copies of your life be deemed beautiful in any acceptable sense of the word? More to the point - what does the math say?

Calculations have been made: the first exact copy of yourself, the calculation goes, is located at some ten to the power ten to the power 29 meters away from you.

But it seems quite wrong. The mistake seems to come from dealing with infinities. At the risk of paraphrasing Richard Feynman who once famously said 'nobody understands quantum mechanics' - nobody quite understands infinities.

The calculation leading to the conclusion that exact copies of you somehow exist in a metaverse is based on the observation that if something, such as yourself, has 
some probability to exist, however small (there is a non-zero probability that you exist in the universe, since you actually do exist), then that probability value multiplied by infinity - the infinity of space-time, of possible events, and indeed separately of both time and spatial extension within an infinite metaverse - yields infinity: ergo, there are infinitely many copies of yourself within the metaverse, and indeed of the world, conditions and environment you live in: QED, quod erat demonstrandum, as the saying goes.

But the non-zero probability that you exist (a probability equal to one, since you do actually exist) has emerged from an infinite pool of probabilities. The incalculable number of micro events and parameters which must come together to eventually yield you is accompanied at every step in the chain of events by randomness stemming from quantum fuzziness. Because the realized outcomes from randomness take their values from an infinite pool of possible outcomes, a layer of infinity exists at every step in the chain of events that eventually produces you.

Hence, the probability to ever produce you again you and your life environment is equal to infinity (representing the infinity of space and time and events in a metaverse) multiplied by the probability for you to emerge from an infinity of possibilities, to wit one divided by the infinity of possible outcomes of the collaborative chain that did eventually produce you once: we are left with infinity divided by infinity, which is an indeterminate number, which could take any value from zero to infinity. There is therefore no mathematical reason why you should ever be produced again within an infinite metaverse at any level, nor is there any reason why someone could not possibly exist again - the only way to assess the mathematical odds would be to try and assess the relative cardinalities of the competing infinities.

Let's illustrate this with a simpler, yet kindred, scenario. Say that you are going to draw the winning numbers of a lottery from a hyperbag containing a full infinity of real numbers. The absolute possibility of drawing any number, say 3345789873578071201 , or 0.00234109 , is not nil: the number exists somewhere within the hyperbag. Yet the probability of drawing that particular number is one divided by ... infinity. It's, in effect, zero. Yet that number was drawn.

(The full picture is actually slightly more complex, as follows: there are two kinds of numbers in the hyperbag: numbers which have a finite digit extension such as, say, 63946254456780177753405 or 2.99335, and numbers which feature an infinite digit extension, such as $\pi$ or $\sqrt{2}$. Any number featuring infinite digit extension could be represented in the hyperbag by the formula that generates it, if that formula exists. Since there is demonstrably an infinity of numbers with finite digit extension (for instance, there is an infinity of prime numbers, which by definition are all finite digit extension numbers), and because including numbers with infinite random extension not representable or generable by a simple formula would give rise to a new category of issues, we'll simply leave out such numbers from the hyperbag. Their absence from the hyperbag does not invalidate the argument.) 
This number draw corresponds to a simpler equivalent of the odds of your being born. Now toss back that number into the hyperbag and keep drawing numbers from the hyperbag for an infinitely long time. There is no guarantee that the first number you drew will ever come back again: the likelihood of that happening is infinity over infinity: it's indeterminate.

Could then these duplicates have to necessarily exist in a higher-level metaverse, say Level 4? Perhaps somewhat more counter-intuitively, (because a Level 4 metaverse is defined as containing everything), the answer is also no.

It still does not work, on at a variety of grounds, the most compelling being that the only universe habitable by anyone human is the level 1 metaverse. Because the laws of physics compatible with human existence are those within the Level 1 metaverse, no human, copy or otherwise, can live anywhere else than in a Level 1 region of any metaverse. If exact copies of yourself do not make it into the Level 1 metaverse, they do not make it anywhere.

There exists an equally compelling, wholly separate reason why there cannot exist such copies of sentient beings anywhere in an infinite multiverse. This reason has to do with time in a three dimensional universe.

Let's consider a near-exact copy of your three dimensional self somewhere in the multiverse. This near-copy is an exact copy of you, except that it will die one second later than you. Another such extant near copy somewhere else one will die 2 seconds later (since there are supposed to be an infinity of copies as well as of all manners of near copies, these particular near-copies must exist too under the infinite-number-ofcopies scenario put forward by Colin Bruce et al.). Another near-copy will die two years later. Another copy will die 20 years later. You see where this is going: in no universe can a near copy of you die, say, six hundred or two thousand years later. Therefore there must necessarily exist, under this proliferated infinities scenario, a tipping point - a precise cut-off point in age count where the probability of anyone still existing tips over from some small finite value to absolute zero. There is indeed definitely a time - at the age mark, say, of 2,000 years old - where the likelihood of anyone being still alive is clearly exactly nil, as well as an age range where the odds of being alive are pretty high, say between 10 and 40 years old. But it is theoretically impossible, on all kinds of compelling grounds, to determine a precise age point where the likelihood of existence suddenly and irrevocably vanishes, i.e. shifts over from a small finite value to nil. This time point would nevertheless have to be specified either within a time quantum interval of time in a discrete time universe, or at a precise cut-off point in a continuous one. For exact and near-exact copies to exist, the copy universe itself would have to be similar to our own at least in the enabling ways that would permit such copies to exist, so that this time point would most likely have to be specified to within Planck time for the scenario to work (i.e., about

$0.000000000000000000000000000000000000000001 \mathrm{sec}$.). But all we can in principle do is estimate a coarse range within which this likelihood becomes nil. 
In an infinite multiverse however, all events have either $100 \%$ or a nil odds of ever being able to exist, irrespective of whether they do.

This probability refers to the possibility that such events may exist, not to their existence per se. For instance, there is a 100\% probability that any given person can possibly like a certain type of music if that person is not deaf, and a $0 \%$ possibility if he or she is deaf. Now the probability of the person actually liking the particular kind of music, rather than being equipped and able to like it, is less than one hundred percent: not everyone who could actually likes it.

A precise point for a switchover between probabilities of $100 \%$ and $0 \%$ cannot in principle be calculated for certain events, which invalidates the notion that such events exist. To put it another way, if there existed copies of yourself that could become slightly older than you, the impossibility in principle of setting an age cut-off point for all such copies would necessarily lead to the existence of copies of yourself reaching impossible ages.

Another possible type of metaverse would be made up of simulated universes. Although this scenario looks like some mad professor's mind figment, its premise is nevertheless sound. In a nutshell, any technical civilization just a tad more advanced than our own would have, in the words of John Barrow (86),

... the capacity to simulate universes in which entities could emerge and communicate with each other, because they would have computer power exceeding ours by a vast factor. Instead of merely simulating their weather or the formation of galaxies, like we do, they would be able to go further and watch the appearance of stars and planetary systems, and, building the laws of biochemistry into their simulations, the evolution of life and consciousness, all speeded up to occur on whatever alien-friendly timescale. Once this ability to simulate universes is achieved, fake universes would proliferate and soon greatly outnumber the real ones.

This is why physicists like Nick Bostrom and Brian Weatherson argue that a thinking being here and now is far more likely to be in a simulated reality than a real one. Could $\mathrm{OM}$ be a software engineer, or perhaps one of a multitude of bored alien teenagers playing a simulation game on his or her very advanced alien computer? We should eventually know: the physicist Martin Savage and his colleagues at the University of Washington say they have devised a sure-fire way whereby the hypothesis that we're living in a simulated Matrix can be tested and decided once and for all.

The notion of a metaverse is sometimes taken to mean a metaverse in the Everett sense, as per his previously cited thesis, the 'Many-Worlds Interpretation of Quantum Mechanics' but as this short and incomplete overview shows, there are many other ways that a multiverse can exist. In the simulated universe scenario, OM would be a software engineer or, indeed perhaps a bored alien teenager, but as soon as we are technically capable of carrying out Martin Savage's test we'll likely demonstrate that this is not who OM is (87).

It's time we looked for OM. 